\title{
PERFIL DEL PACIENTE HIPERTENSO CON SOSPECHA CLÍNICA DE APNEA OBSTRUCTIVA DEL SUEÑO EN EL CENTRO DE DIAGNÓSTICOY MEDICINA AVANZADA Y DE CONFERENCIAS MÉDICAS Y TELEMEDICINA (CEDIMAT) DURANTE EL PERÍODO ENERO 2019 - FEBRERO 2020, SANTO DOMINGO, REPÚBLICA DOMINICANA
}

\section{Profile of the hypertensive patient with clinical suspicion of obstructive sleep apnea at the Center for Diagnosis and Advanced Medicine and Medical Conferences and Telemedicine (CEDIMAT) during the period January 2019 - February 2020, Santo Domingo, Dominican Republic}

\section{Jesús Antonio Echavarría Uceta ${ }^{\mathrm{y}}$ Hector Herrera Mercedes ${ }^{\mathrm{b}}$}

Recibido: 6 de julio, $2020 \bullet$ Aprobado: 12 de octubre, 2020

Cómo citar: Echavarría Uceta JA, Herrera Mercedes H. Perfil del paciente hipertenso con sospecha clínica de apnea obstructiva del sueño en el Centro de Diagnóstico y Medicina Avanzada y de Conferencias Médicas y Telemedicina (CEDIMAT) durante el período enero 2019 - febrero 2020, Santo Domingo, República Dominicana. cysa [Internet]. 10 de junio de 2021 [citado 16 de junio de 2021];5(2):95-100. Disponible en: https://revistas.intec.edu.do/index.php/cisa/article/view/2222

\section{Resumen}

Introducción: la apnea obstructiva del sueño es una patología respiratoria caracterizada por paradas de la respiración de forma repetida durante el sueño. Esta patología está relacionada con los trastornos hipertensivos, pudiendo agravarlos.

Objetivo: determinar el perfil de un paciente hipertenso bajo sospecha clínica de apnea obstructiva del sueño en el Centro de Diagnóstico y Medicina Avanzada y de Conferencias Médicas y Telemedicina (CEDIMAT) durante el periodo enero 2019 - febrero 2020.

Material y métodos: se realizó un estudio observacional-descriptivo, transversal con datos retrospectivos. Se estudiaron 38 expedientes clínicos de pacientes hipertensos de la consulta de neumología y que cumplen con criterios de sospecha clínica de apnea obstructiva del sueño.

\begin{abstract}
Introduction: Obstructive sleep apnea is a respiratory disease characterized by repeated stops of breathing during sleep. This pathology is related to hypertensive disorders, which can aggravate them.
\end{abstract}

Objective: To determine the profile of a hypertensive patient under clinical suspicion of obstructive sleep apnea at the Center for Diagnosis and Advanced Medicine and Medical and Telemedicine Conferences (CEDIMAT) during the period January 2019 - February 2020.

Material and methods: An observational study of descriptive, cross-sectional type with retrospective data. Thirty-eight clinical records of hypertensive patients from the pulmonology clinic and who meet the criteria for clinical suspicion of obstructive sleep apnea were studied.

\footnotetext{
b Universidad Iberoamericana, UNIBE, ORCID: 0000-0002-5642-6292, Correo-e: hherrera1@est.unibe.edu.do
}

a Universidad Iberoamericana, UNIBE, ORCID: 0000-0001-9611-478X, Correo-e: jechavarria1@est.unibe.edu.do 
Resultados: el rango de edad fue de 40-64 años con un $55.26 \%$. El $66 \%$ son masculinos. $36.84 \%$ son hipertensos grado I mientras que otro $34.21 \%$ tienen sus niveles tensionales normales. El $68.41 \%$ son obesos y un $26.32 \%$ están en sobrepeso. $63.16 \%$ se clasifican como IV en la escala de Mallampati.

Conclusión: el perfil de los pacientes hipertensos bajo sospecha clínica de apnea obstructiva del sueño es: sexo masculino, adultez media, obesidad, escala de mallampati categoría IV y el uso de múltiples fármacos en el control de la hipertensión.

Palabras clave: perfil de salud; síndrome de la Apnea del Sueño; hipertensión; obesidad; Índice de Masa Corporal.

\section{Introducción}

La apnea obstructiva del sueño, también definida como síndrome de apneas-hipoapneas obstructivas del sueño (SAHOS), constituye un trastorno respiratorio crónico de gran relevancia en la población general $^{1}$. Es una patología que afecta tanto el bienestar físico como mental del individuo que la padece. Se caracteriza por episodios recurrentes de obstrucción completa (apnea) o parcial (hipopnea) de las vías respiratorias superiores que provocan hipoxemia e hipercapnia intermitentes, microdespertares corticales, aumento del estrés oxidativo y fragmentación del sueño ${ }^{2}$.

El SAHOS es un trastorno altamente frecuente que afecta del $2 \%$ al $4 \%$ en mujeres y a los hombres entre el $4 \%$ al $6 \%$ de la población adulta a nivel mundial ${ }^{3}$. Se estima que la mayoría de los adultos con SAHOS moderado-severo no están diagnosticados. La revisión sistemática de la Agency for Healthcare Research and Quality (AHRQ) publicada en $2017^{4}$ revela, en población general, una prevalencia del $10 \%$ para SAHS moderado, creciente con la edad $^{5}$. En EE. UU. se menciona que este síndrome afecta al 4-24\% de los hombres y al 2-9\% de las mujeres y se estima que al menos $20 \%$ de los adultos
Results: The age range was 40-64 years with $55.26 \% .66 \%$ are male. $36.84 \%$ are grade I hypertensive while another $34.21 \%$ have normal blood pressure levels. $68.41 \%$ are obese and $26.32 \%$ are overweight. $63.16 \%$ are classified as IV on the mallampati scale.

Conclusion: The profile of hypertensive patients under clinical suspicion of obstructive sleep apnea is; male sex, middle adulthood, obesity, category IV of mallampati scale and the use of multiple drugs in the control of hypertension.

Keywords: Health Profile; Sleep Apnea Syndrome; hypertension; obesity; Body Mass Index.

de edad media tienen SAHOS leve y que el $80 \%$ de los casos permanecen sin diagnosticar ${ }^{6}$.

Los efectos adversos que trae consigo el SAHOS son importantes mediadores del riesgo cardiovascular $^{7}$, causan elevaciones temporales de la presión arterial en asociación con la desaturación de oxígeno en sangre, la excitación y la activación simpática y pueden causar presión arterial elevada durante el día $y$, en última instancia, hipertensión sostenida ${ }^{8}$.

La Hipertensión Arterial es un trastorno que golpea de manera abrumadora a nivel mundial y es aún más letal en países de bajos y medianos ingresos. Informes recientes de la Organización Mundial de la Salud (OMS) y del Banco Mundial destacan la importancia de las enfermedades crónicas tales como la hipertensión, como obstáculo al logro de un buen estado de salud?

Hace medio siglo se desconocía el SAHOS y por consecuencia su impacto. Sin embargo, durante las últimas décadas su relevancia es evidente debido a que representa una causa significativa de morbilidad y mortalidad ${ }^{10}$. La coexistencia entre la AOS y la HTA está bien establecida y el trastorno respiratorio es una de las causas más comunes de la forma secundaria del estado hipertensivo ${ }^{11}$. 
El síndrome de apnea-hipopnea obstructiva del sueño (SAHOS) necesita una evaluación médica completa y requiere estudios paraclínicos para confirmar su presencia, su grado de severidad y estados comórbidos, el estudio por excelencia de acuerdo a los niveles de recomendación de la Academia Americana de Medicina del Sueño (AASM, por sus siglas en inglés) es la Polisomnografía basal ${ }^{12,13}$.

Sin embargo, en República Dominicana la Polisomnografía basal es un estudio todavía limitado por accesibilidad y situación económica, no obstante, la triada clínica principal: Roncopatía crónica, Apneas presenciadas e Hipersomnia diurna o tendencia a dormirse involuntariamente ante situaciones inapropiadas ${ }^{14}$ nos guían hacia una sospecha de la enfermedad.

\section{Material y métodos}

Este estudio es de tipo observacional descriptivo, transversal con datos retrospectivos, donde se buscó determinar un perfil de los pacientes hipertensos bajo sospecha clínica de apnea obstructiva del sueño en el Centro de Diagnóstico y Medicina Avanzada y de Conferencias Médicas y Telemedicina (CEDIMAT). Dicho estudio se llevó a cabo en CEDIMAT, en Santo Domingo, República Dominicana, durante el periodo enero 2019 - febrero 2020.

La recolección de datos de esta investigación fue a través de expedientes digitalizados del Centro de Diagnóstico y Medicina Avanzada y de Conferencias Médicas y Telemedicina (CEDIMAT) y en estos las variables observadas para delimitar el perfil fueron la edad, el sexo, el estado hipertensivo del paciente, el índice de masa corporal, la circunferencia cervical, las comorbilidades del paciente, los hábitos tóxicos, el grado según la escala de mallampati en el cual se encontraban los pacientes y cantidad de fármacos que utilizan para el control de su trastorno hipertensivo.

Los criterios de elección para la muestra se basaron en pacientes entre los 20 y 90 años que acudían a la consulta de neumología, los cuales ya habían sido diagnosticados con hipertensión y que a su vez se encontraban bajo sospecha clínica de síndrome de apnea obstructiva del sueño. La muestra estuvo compuesta por 38 pacientes.

Se respetaron los datos personales de los pacientes seleccionados para esta investigación, garantizando la confidencialidad y no divulgación de ningún antecedente y dato personal de cada uno de los pacientes. Este estudio fue aprobado por el Comité de Ética y el Decanato de Investigación Académica de la Universidad Iberoamericana UNIBE, previo al levantamiento de datos.

Después de recolectar los datos, estos se almacenaron en Microsoft Excel, analizaron tomando en cuentas las variables planteadas para así determinar un perfil generalizado de un paciente hipertenso bajo sospecha clínica de apnea obstructiva del sueño.

\section{Resultados}

Durante el periodo de estudio, enero 2019 - febrero 2020, en el Centro de Diagnóstico y Medicina Avanzada y de Conferencias Medicas y Telemedicina (CEDIMAT) se investigaron 38 expedientes digitalizados.

Tabla 1. Características Sociodemográficas: Distribución según rangos de edad en pacientes hipertensos bajo sospecha clínica de apnea obstructiva del sueño

\begin{tabular}{|l|l|l|}
\hline EDAD & Frecuencia & Porcentaje \\
\hline Adultez (40-64 años) & 21 & $55.26 \%$ \\
\hline Adultez Temprana (20-39 años) & 2 & $5.26 \%$ \\
\hline Tercera edad (65-90 años) & 15 & $39.47 \%$ \\
\hline TOTAL & $\mathbf{3 8}$ & $\mathbf{1 0 0 . 0 0} \%$ \\
\hline
\end{tabular}

Fuente: análisis de la base de datos del estudio. 
Un $36.84 \%$ de los pacientes son hipertensos de primer grado, $13.16 \%$ hipertensos grado II y $7.89 \%$ con presión elevada, respectivamente.

Un $31.58 \%$ de los pacientes tiene una circunferencia cervical mayor o igual a $41 \mathrm{~cm}$ y un $13.16 \%$ con una circunferencia cervical menor o igual a $40 \mathrm{~cm}$.

Tabla 2. Distribución según IMC en pacientes hipertensos bajo sospecha clínica de apnea obstructiva del sueńo

\begin{tabular}{|l|l|l|}
\hline CLASIFICACIÓN IMC & Frecuencia & Porcentaje \\
\hline Normal & 2 & $5.26 \%$ \\
\hline Obesidad I & 9 & $23.68 \%$ \\
\hline Obesidad II & 9 & $23.68 \%$ \\
\hline Obesidad III & 8 & $21.05 \%$ \\
\hline Sobrepeso & 10 & $26.32 \%$ \\
\hline TOTAL & $\mathbf{3 8}$ & $\mathbf{1 0 0 . 0 0} \%$ \\
\hline
\end{tabular}

Fuente: análisis de la base de datos del estudio.

Un 36.84\% padecen de enfermedades endocrinológicas (diabetes mellitus 2 y trastornos tiroideos), $21,05 \%$ padecen de enfermedades respiratorias (asma bronquial y rinosinusitis crónica).

Tabla 3. Distribución según Escala de Mallampati en pacientes hipertensos bajo sospecha clínica de apnea obstructiva del sueño

\begin{tabular}{|l|l|l|}
\hline MALLAMPATI & Frecuencia & Porcentaje \\
\hline II & 3 & $7.89 \%$ \\
\hline III & 8 & $21.05 \%$ \\
\hline IV & 24 & $63.16 \%$ \\
\hline No Disponible & 3 & $7.89 \%$ \\
\hline TOTAL & $\mathbf{3 8}$ & $\mathbf{1 0 0 . 0 0} \%$ \\
\hline
\end{tabular}

Fuente: análisis de la base de datos del estudio.
Un $26.32 \%$ fuma cigarrillos, un $13.16 \%$ toma café y un $10.53 \%$ consume alcohol. Un $52.63 \%$ utilizan más de un fármaco y un $34.21 \%$ utilizan uno.

Tabla 4. Perfil del paciente hipertenso con sospecha clínica de apnea obstructiva del sueño

\begin{tabular}{|l|l|}
\hline Sexo & Masculino \\
\hline Edad & Adultez Media \\
\hline IMC & Hipertensión Grado 1 \\
\hline Circunferencia Cervical & $\begin{array}{l}\text { Obesidad } \\
41 \mathrm{~cm}\end{array}$ \\
\hline Escala de Mallampati & VI \\
\hline Comorbilidad & $\begin{array}{l}\text { Enfermedades Endocri- } \\
\text { nológicas }\end{array}$ \\
\hline Hábitos Tóxicos & No Conocido \\
\hline Medicamentos & $\begin{array}{l}\text { Mas de 2 medicamentos } \\
\text { para controlar hiperten- } \\
\text { sión arterial. }\end{array}$ \\
\hline
\end{tabular}

Fuente: análisis de la base de datos del estudio.

\section{Discusión}

Los datos sociodemográficos en este estudio (edad y sexo) fueron similares con el estudio de Fátima Cintra et al. en Brasil, donde la edad promedio fue de $52,03 \pm 9$ años y predomino el sexo masculino con un $54,4 \%{ }^{15}$.

En cuanto a la variable del índice de masa corporal y la circunferencia cervical en nuestra investigación encontramos valores por encima de la normalidad en ambas variables, estos resultados se asemejan a los de la investigación de Liza María González Hernández et al., intitulada Relación entre hipertensión arterial sistémica y sindrome de apnea-hipopnea obstructiva del sueño y sus factores de riesgo asociados en Cali, Colombia donde muestran un promedio de IMC de $30 \pm 6.1$ y un promedio de circunferencia cervical de $41 \pm 5,3^{16}$. 
En nuestro estudio se evidenció con mayor porcentaje, pacientes con hipertensión de primer grado. Estos datos coinciden a su vez con los resultados de Fátima Cintra et al., donde en promedio los pacientes con Apnea Obstructiva del Sueño son Hipertensos Grado I ${ }^{15}$.

A partir de las características estudiadas de la muestra en esta investigación se puede sugerir un perfil para los pacientes hipertensos bajo sospecha clínica de apnea obstructiva del sueño; es un paciente masculino, de adultez media, hipertenso de primer grado, obeso con una circunferencia cervical mayor a $41 \mathrm{~cm}$, con una escala de Mallampati categoría VI, con enfermedades endocrinológicas donde prevalece la diabetes, sin hábitos tóxicos conocidos, y que utilizan más de 2 medicamentos para tratar su hipertensión.

Es vital que la comunidad científica se concientice de la importancia de la enfermedad, de igual manera que se eduque al personal de salud y a la comunidad en general para lograr una adecuada detección de la enfermedad.

\section{Bibliografía}

1. Blanco M, Ernst G, Valiensi S, Berrozpe E, Martínez O, Borsini E. Eficiencia Del sueño en la polisomnografía nivel II de pacientes ambulatorios y hospitalizados. MEDICINA (Buenos Aires). 2020;80:317-23.

2. Mokhlesi B, A. Finn L, W. Hagen E, Young T, Mae Hla K, Van Cauter E and E. Peppard P. Obstructive sleep apnea during REM sleep and hypertension. Results of the Wisconsin sleep cohort. 2014;190(10):1158-67.

3. Durán J, Esnaola S, Rubio R, Iztueta A. Obstructive sleep apnea-hypopnea and related clinical features in a population-based sample of subjects aged 30 to 70 yr. American Journal of Respiratory and Critical Care Medicine 2001;163(3 Pt 1):685-9.
4. Jonas D, Amick H, Feltner C, et al. Screening for obstructive sleep apnea in adults JAMA. 2017;317(4):415-33.

5. Cruces C, Herves C, Martin V, Hernaiz S, Lago I, Montero M, Orge M, Roca J, Claveria A, Utilidad diagnóstica del cuestionario STOPBang en la apnea del sueño moderada en atención primaria. Gaceta Sanitaria. 2019;33(5):421-6.

6. Hidalgo-Martínez P, Lobelo R. Epidemiología mundial, latinoamericana y colombiana y mortalidad del síndrome de apnea-hipopnea obstructiva del sueño (SAHOS). Revista de la facultad de medicina. 2017;65:S17-20.

7. Somers V, White D, Amin R, Abraham W, Costa F, Culebras A, Daniels S, Floras J, Hunt C, Olson L, Pickering T, Russell R, Woo M, y Young T. Circulation. 2008;118(10):1080-111.

8. Peppard P, Young T, Palta M, Skatrud J. Prospective study of the association between sleep-disordered breathing and hypertension. The New England Journal of Medicine. 2000; 342:1378-84.

9. Sánchez R, Ayala M, Baglivo H, Velázquez C, Burlando G, Kohlmann O, Jiménez J, Jaramillo P, Brandao A, Valdés G, Alcocer L, Bendersky M, Ramirez A y Zanchetti A. Guías Latinoamericanas de Hipertensión Arterial. Revista Chilena de Cardiología. 2010;29:117-44.

10. Blanhir J, Valencia-Flores M, y Lozano-Cruz O. El síndrome de apnea obstructiva del sueño como factor de riesgo para enfermedades cardiovasculares y su asociación con hipertensión pulmonar. Neumología y cirugía de tórax. 2017;76(1): 51-60.

11. Rimoldi SF, Scherrer U, Messerli FH. Secondary arterial hypertension: when, who, and how to screen? European Heart Journal. 2014;35 (19):1245-54. 
12. Kapur V, Auckley D, Chowdhuri S, et al. Clinical practice guideline for diagnostic testing for adult obstructive sleep apnea: an American Academy of Sleep Medicine Clinical Practice Guideline JAMA. Journal of Clinical Sleep Medicine. 2017;13(3):479-504.

13. Venegas-Mariño M y Franco-Vélez A. Métodos diagnósticos en el síndrome de apnea-hipopnea obstructiva del sueño (SAHOS). Revista de la Facultad de Medicina. 2017;65(1):91-5.

14. Páez-Moya S Y Parejo-Gallardo K. Cuadro clínico del síndrome de apnea-hipopnea obstructiva del sueño (SAHOS). Revista de la Facultad de Medicina. 2017;65:29-37.
15. Cintra F, Tufik S, Paola F, Melo-Fujita L, Oliveira W, et al . Perfil cardiovascular en pacientes con apnea obstructiva del sueño. Arquivos Brasileiros de Cardiologia 2011;96(4):293-9.

16. González L, Castaño J, Herrera V, Jiménez AM, Hoyos P, Ramírez A, Zuluaga LM. Relación entre hipertensión arterial sistémica y síndrome de apnea-hipopnea obstructiva del sueño y sus factores de riesgo asociados, en población hipertensa de un centro médico. Archivos de Medicina (Col). 2008;8(2):89-97. 